\title{
Hygiene im OP
}

\section{Volkmar Heppert, Alfons Martens, Andreas Wentzensen}

\section{Zusammenfassung}

Jede postoperative Wundinfektion bedeutet für den Patienten eine schwere Komplikation, die sein/ihr weiteres Leben entscheidend beeinflussen kann. Für die Versicherungsträger erzeugt ein Infektverlauf eine erhebliche finanzielle Mehrbelastung. Zunehmend sind Tendenzen nachweisbar, diese Mehrbelastung auf den vermeintlichen „Verursacher“, die Klinik, abzuwälzen. Das DRG-System unter- stützt dies dahingehend, als der Infekt, der innerhalb der „Oberen Grenzverweildauer" (OGD) auftritt und zur operativen Revision zwingt, nicht zusätzlich vergütet wird. Die Mehrkosten bedeuten damit für die erstbehandelnde Klinik eine erhebliche finanzielle Belastung. Dies muss dazu führen, dass dem Standard der Hygiene die wesentliche Schlüsselstellung zukommt, derartige Infektionen zu minimieren.

\section{Einleitung}

Unter nosokomialen Infektionen versteht man die im Krankenhaus erworbenen Infektionen. Der postoperative Wundinfekt ist die häufigste Form. Obwohl die so genannte NIDEP-Studie von 1997 in kritischer Diskussion stand, konnte sie doch klar belegen, dass immerhin 30\% dieser Infektionen vermeidbar sind. Die finanzielle Belastung des Gesamtsystems wird ersichtlich, wenn man bedenkt dass laut Literatur - und da gehen die Zahlen weit auseinander - jede postoperative Infektion Mehrkosten zwischen $15000 €$ und $100000 €$ verursacht.

Die NIDEP-Studie hat dies zahlenmäßig klar unterstrichen. Die Publikation des Bundesministeriums für Gesundheit Band 126 hat leider mehr auf diese „Zahlenspiele“ als auf die Betroffenheit der Patienten Wert gelegt. Jeder postoperative Wundinfekt verlängert nämlich den stationären Aufenthalt für den Patienten um durchschnittlich 7,3 Tage.

Uns, die wir in der Klinik, quasi „vor Ort“ tätig sind, muss es ein unverzichtbares

OP-JOURNAL 2005; 21: 68-73

(c) Georg Thieme Verlag KG Stuttgart · New York
Anliegen sein, durch adäquate Technik, modernste Operationsverfahren, aber vor allem durch unsere eigene Hygienedisziplin dazu beizutragen, die Infektrate so niedrig wie möglich zu halten. Gerade unter den Kostengesichtspunkten des Systems der „Diagnosis Related Groups“ (DRG) erhält die Hygiene eine neue Brisanz. Wie viel ist nötig? Wie viel können wir uns leisten? Gegenüber den Verwaltungen muss hier eine klare, nachvollziehbare Strategie erarbeitet werden, die es uns ermöglicht, gerade jetzt, wo Sparen angesagt erscheint auch weiterhin den Standard hochzuhalten.

Die Hygienemaßnahmen in den operativen Fächern müssen nämlich 3 Ziele verfolgen:

- Die grundsätzliche Vermeidung der nosokomialen Infektion.

- Die Vermeidung der Kreuzkontamination hat unter Methicillin Resistentem Staphylococcus aureus (MRSA)- und Vancomycin Resistenten Enterokokken (VRE)-Gesichtspunkten deutlich an Brisanz gewonnen.

- Die Vermeidung der eigenen Infektion. Zunehmend wird dies ein Problem in der Zukunft sein können. In Zeiten von Hepatitis (grundsätzlich unterschätzt), AIDS (publizistisch im Vordergrund stehend aber mit viel geringerer Fallzahl als die Hepatitis) und MRSA (was passiert mit nicht sanierbarem kontaminierten Personal) kommt dem Schutz des Personals gleiche Bedeutung zu, wie dem Schutz des Patienten.

\section{Hauptteil}

\section{Operationstechnik}

Die höchste Bedeutung auf den Einfluss der Wundinfektion hat das sichere gewebeschonende und standardisierte Operieren. Obwohl ca. 40\% aller Wunden als kontaminiert angesehen werden müssen, Routineabstriche vor Hautnaht bei aseptischen Eingriffen haben dies gezeigt, liegen die Infektionsraten der modernen Traumatologie „nur" bei 2-3\%. Auch bei offenen Frakturen, die einen Kontaminationsgrad von über $50 \%$ zeigen, liegt das wirkliche Infektrisiko unter $10 \%$. Das sichere Wunddebridement, die biologischen Osteosynthesetechniken und neue Erkenntnisse der Wundheilung haben vermutlich mehr zur Senkung der Infektraten beigetragen, als viele bauliche und finanziell aufwändige Hygienemaßnahmen.

Dennoch bleibt die Infektion eine permanente Bedrohung. Ein im Januar 1997 geschaffenes Krankenhaus-Infektions-Surveillance-System (KISS) dient der Überwachung von Infektionsraten bei definierten Referenzoperationen. Nur Kliniken mit mindestens 30 Operationen durften sich an dieser kontinuierlich weiterlaufenden Studie beteiligen. Die Ergebnisse können unter der Internetseite

http://www.nrz-hygiene.de/dwnld/referenz_op_pdf

abgefragt werden. Das so genannte Hawthorne-Prinzip, bei dem sich unter kontinuierlicher Beobachtung die Arbeitsqualität verbessern soll, konnte hier leider nicht wirklich verwirklicht werden. Die Infektraten der einzelnen Referenzoperationen sind seit 1997 praktisch gleich 
geblieben, obwohl die dort aufgeführten Operationen weitest gehend standardisiert verlaufen.

Somit müssen andere Einflussfaktoren auf die Entstehung der Infektion diskutiert werden. Bei vielen dieser Faktoren spielt die Hygienedisziplin der beteiligten Personen eine wesentliche Rolle.

\section{Händedesinfektion}

Der Händedesinfektion kommt eine wesentliche Schlüsselstellung bei der Übertragung nosokomialer Infektionen zu. Mehr als 90\% werden nachweislich über die Hände übertragen [1]. Gerade im OP kommt dieser Aufgabe eine zunehmend wichtigere Bedeutung zu. So empfiehlt das Robert-Koch-Institut (RKI):

Die chirurgische Händedesinfektion ist vor allen operativen Eingriffen durchzuführen. Bedingungen sind:

- Fingernägel müssen kurz und rund geschnitten sein.

- Es dürfen keine Nagelbettverletzungen oder entzündliche Prozesse vorhanden sein.

- Ausschließlich Nägel und Nagelfalze sollen bei Bedarf mit weicher (!), (thermisch) desinfizierter Kunststoffbürste und hygienischem Handwaschpräparat gereinigt werden.

- Bürsten der Hände und Unterarme ist wegen Hautirritation und höherer Keimabgabe zu unterlassen.

- Armaturen und Spender dürfen nicht über Handkontakt bedient werden!

Vor der am OP-Tag erstmalig durchgeführten chirurgischen Händedesinfektion werden Hände und Unterarme bis zum Ellenbogen mit nach oben gerichteten Fingerspitzen und tief liegendem Ellenbogen während etwa 1 min mit einem Handwaschpräparat gewaschen.

Länger dauernde Händewaschungen sind wegen potenzieller Hautschädigung abzulehnen, zumal dadurch keine weitere Keimzahlverminderung erreicht wird. Nach Abtrocknen mit einem keimarmen Einmalhandtuch wird die Händedesinfektion durchgeführt.

Während der vom Hersteller der Präparate angegebenen Einwirkungszeit müssen Hände und Unterarme vollständig mit Desinfektionslösung benetzt sein. Eine Händetrocknung danach ist mit Rekontaminationsrisiko verbunden und nicht erforderlich (falls jedoch gewünscht, nur mit sterilem Tuch zur Einmalbenutzung); allerdings müssen die Hände vor dem Anlegen der OP-Handschuhe lufttrocken sein, um Hautschäden vorzubeugen und die Integrität des OP-Handschuhs nicht zu gefährden. Bei Aufeinanderfolge kurzer Eingriffe (OP + OP-Pause $<60 \mathrm{~min}$ ) mit geringer Kontaminationswahrscheinlichkeit (intakter Handschuh!) kann vor dem nächsten Eingriff die Händewaschung unterbleiben.

Ferner sind folgende Aspekte von Bedeutung:

Durch Einbürsten eines alkoholischen Desinfektionsmittels in den Nagelfalz kann eine Wirkungssteigerung erzielt werden; dies empfiehlt sich, wenn eine hohe Keimarmut erforderlich ist, z.B. vor Implantation alloplastischer Materialien.

Sofern bei der Händewaschung bereits die OP-Bereichskleidung angelegt ist, sollte eine wasserundurchlässige keimarme Schürze getragen werden, um ein Durchnässen der OP-Bereichskleidung zu verhindern.

Es ist darauf $\mathrm{zu}$ achten, dass Bereiche oberhalb des Ellenbogens (Ärmel!) nicht befeuchtet werden.

Hautpflege an Händen und Unterarmen ist eine berufliche Pflicht, weil bereits kleinste Risse bzw. Mikrotraumen potenzielle Erregerreservoire sind und sich eine nicht gepflegte Haut nicht sicher desinfizieren lässt [2],

Auch Hautpflege ist Pflicht. Allerdings müssen dafür vom Klinikträger auch entsprechende Spender an den wesentlichen Bedarfspunkten vorgehalten werden. Ein entsprechender Hautschutzplan ist klinikweit auszuhängen und im Hygieneordner zu hinterlegen.

Um größtmögliche Sicherheit zu gewährleisten, ist es unserer Meinung nach unverzichtbar, mit Beginn der Händedesinfektion eine Uhr - muss ebenso wie ein Spiegel zur optischen Kontrolle des Mundschutzes vorgehalten werden - zu stellen. Die im Hygieneplan vorgeschrieben Zeit der Händedesinfektion muss unbedingt eingehalten werden. Kittel und Handschuhe werden erst angezogen, wenn die Hände abgetrocknet sind, da die Einwirkung von Desinfektionsmitteln die OP-Handschuhe porös werden lässt. Die Schutzfunktion für Arzt und Patient wird gestört.
Der zunehmenden Verkürzung der Desinfektionszeit (1,5 min) von Seiten der Industrie ist nicht unbedingt Folge zu leisten, auch wenn dabei unter DRG-Gesichtspunkten scheinbar Zeit gespart werden kann. Die Disziplin ist leider nicht so gut entwickelt, dass die vorgegebene Zeit immer eingehalten wird. Hier muss grundsätzlich und unabhängig von der Position in der Hierarchie von Seiten der im OP-Arbeitenden jeder Missstand offen angesprochen werden. Gerade die Tatsache, dass diese Offenheit nicht besteht, ist kontraproduktiv und dient nicht der Hygienedisziplin. Andererseits ist natürlich zu fordern, dass diese Hygienedisziplin natürlich in der Hierarchie von „oben“ nach „unten“ vorgelebt werden muss. Wie will man Mitarbeiter integrieren, wenn die Führungspersonen sich Ausnahmen zugestehen würden?

\section{Hautdesinfektion}

Eine sterile Hautoberfläche wird es bei Patienten nie geben. Das Ziel der Hautdesinfektion ist daher die weitest gehende Vernichtung von transienter und residenter Hautflora. Dies kann aber nur dann gelingen, wenn ausschließlich DGHM-gelistete und zugelassene Präparate Verwendung finden (siehe Abb. 1). Die erforderliche Einwirkzeit ist dabei zu beachten. Es wird unterschieden zwischen talgdrüsenreicher und talgdrüsenarmer Haut (siehe Abb. 2), wobei sich die geforderten Zeiten dabei erheblich unterscheiden (Richtlinie Krankenhaushygiene 2001. Lieferung 17.).

Geforderte Einwirkzeit von Hautdesinfektionsmitteln

Talgdrüsenarme Haut $15 \mathrm{~s}-1$ min Talgdrüsenreiche Haut $>10$ min

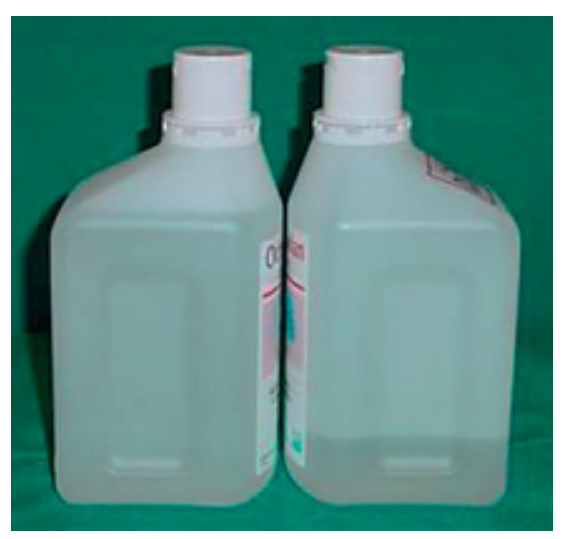

Abb.1 Zum Verwechseln ähnlich, aber nur eines der Präparate ist zur chirurgischen Hautdesinfektion zugelassen. 


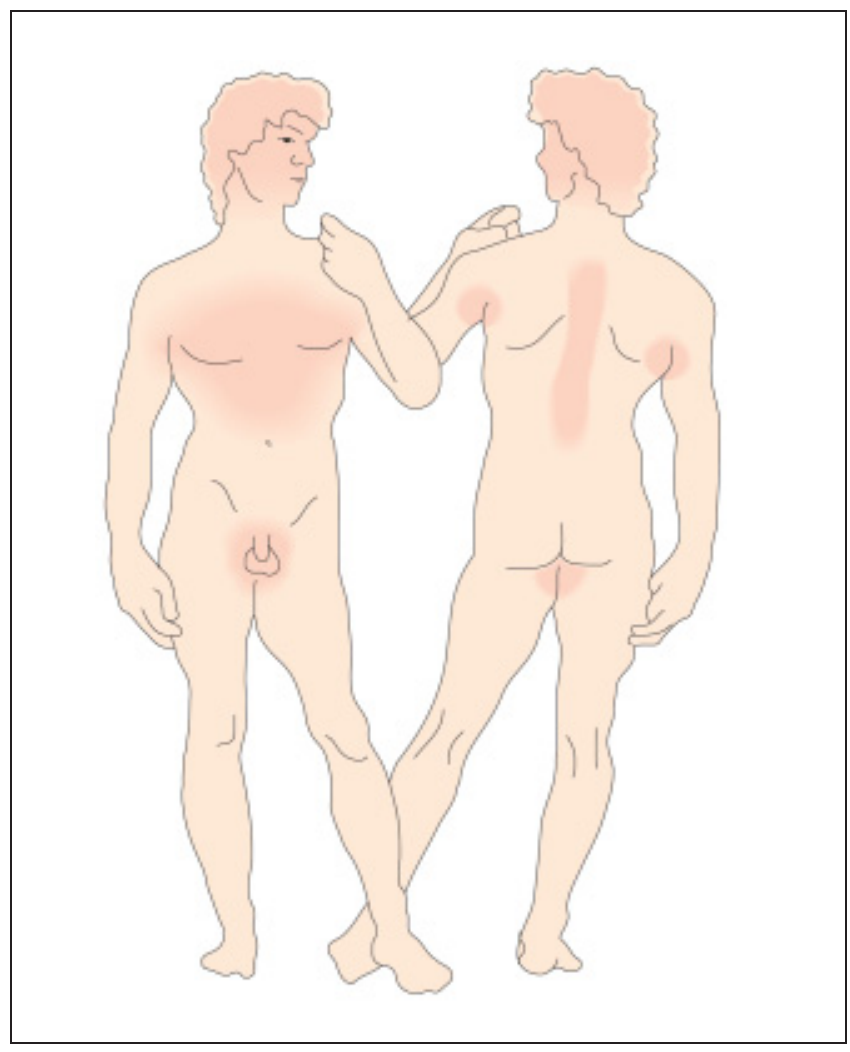

Grundsätzlich ist ausschließlich eine Reduktion der Keimflora möglich. Zu unterscheiden ist zwischen den einzelnen zugelassenen Präparategruppen. Jedes hat seine Vor- und Nachteile (siehe Abb. $\mathbf{3}$ ):

- Chlorhexidin wird dabei nicht inaktiviert von Blut und Serumproteinen

- Chlorhexidin: Keimreduktion Faktor 9

- PVP Jod hat im Vergleich aber einen konstant bakteriostatischen Effekt, solange es auf der Haut ist

- Povidon-Iod: Keimreduktion Faktor $1,3-1,9$
Abb. 2 Verteilung von talgdrüsenarmer und -reicher Haut. Beachte die unterschiedlichen Einwirkzeiten It. Richtlinie Krankenhaushygiene 2001.
Schmuck

Dies ist ein leidiges Thema, welches immer wieder zu Diskussionen führt. Aber dies ergibt keinen Sinn und sollte auch so nicht akzeptiert werden. Anzuführen ist hier einerseits die Unfallverhütungsvorschrift (UVV). Ungeachtet hygienischer Bedenken gilt laut §44 UVV:

„In Arbeitsbereichen mit erhöhter Infektionsgefährdung dürfen an Händen und Unterarmen keine Schmuckstücke, Uhren und Eheringe getragen werden.“

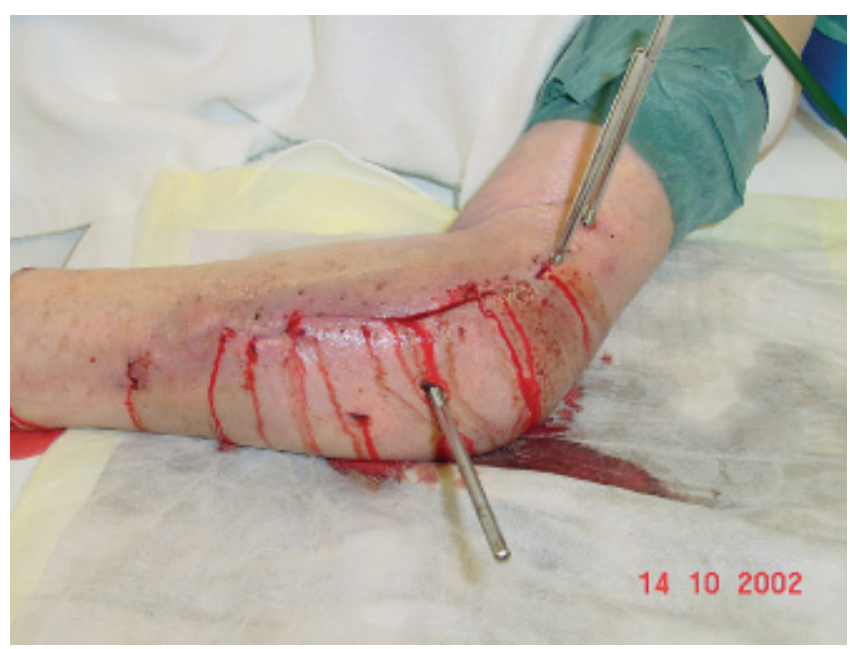

Abb. 3 Geplanter Verfahrenswechsel auf interne Osteosynthese. Blut verhindert die Desinfektion der Haut, die unter dem Blutfilm sich befindet.
Tab. 1 Keimbesiedelung der Hände beim Tragen von Schmuck nach Händedesinfektion

\begin{tabular}{lll} 
& mit Ring & ohne Ring \\
\hline $\begin{array}{l}\text { Gram. neg. } \\
\text { Stäbchen }\end{array}$ & $25 \%$ & $9,6 \%$ \\
$\begin{array}{l}\text { Staph. aureus } \\
\text { Candida }\end{array}$ & $25 \%$ & $9,5 \%$ \\
& $21 \%$ & $8,6 \%$
\end{tabular}

Tab. 2 Mittlere Keimzahl pro Hand nach Händedesinfektion

\begin{tabular}{lll} 
& mit Ring & ohne Ring \\
\hline $\begin{array}{l}\text { Gram. neg. } \\
\begin{array}{l}\text { Stäbchen } \\
\text { Candida }\end{array}\end{array}$ & 325 & 50 \\
& 120 & 10
\end{tabular}

Tab. 3 Keimbesiedelung der Hände nach Händedesinfektion

\begin{tabular}{ll} 
Kein Ring & $29 \%$ \\
\hline 1 Ring & $76 \%$ \\
2 Ringe & $94 \%$
\end{tabular}

Unabhängig davon gibt es auch klare hygienische Gründe, dies im Operationsbereich nicht zu tolerieren (Tab. 1 -3). Mehrere Studien konnten klar belegen, dass durch das Tragen von Schmuck im OP trotz Händedesinfektion Keimbelastungen nachweisbar waren, die nicht akzeptiert werden dürfen [4]. Wir weisen darauf hin, dass z. B. in der Chipindustrie extra sterile Reinräume geschaffen wurden, damit die Leistungsfähigkeit der Unterhaltungselektronik störungsfrei funktioniert. Wir, die Kunden wären erbost, wenn der „Game Boy“ einmal nicht funktioniert. Aber wir bauen in die Patienten Implantate ein. Müssen wir dann nicht höhere Sterilitätskriterien festschreiben, als bei der Unterhaltungselektronik? Wir sind dazu verpflichtet.

UVV §44: „In Arbeitsbereichen mit erhöhter Infektionsgefährdung dürfen an Händen und Unterarmen keine Schmuckstücke, Uhren und Eheringe getragen werden."

\section{Präoperative Rasur}

Unter zunehmendem Kostendruck muss der Umsatz pro Zeit in der Klinik deutlich ansteigen. Allein schon diese Formulierung, entnommen aus der Wirtschaftswissenschaft, ist schwierig. Ungeachtet dessen muss er hinterfragt werden, da 


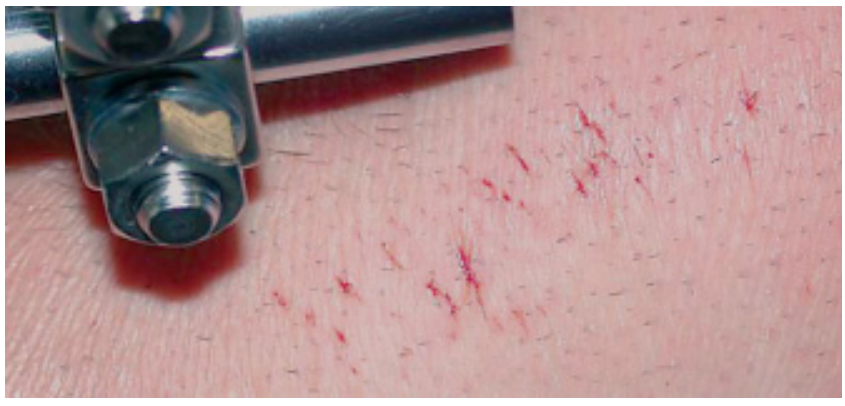

Abb. 4 Kleinste Hautwunden nach einer Hautrasur bedeuten ein deutlich erhöhtes Infektrisiko für den Patienten. wir es doch mit Menschen zu tun haben. Aber leider ist dies mittlerweile klinische Realität geworden. Dies ist unangenehm, aber wir können uns dem nicht entziehen.

Die einzelnen Funktionsbereiche in den Kliniken versuchen nachvollziehbar, die Mehrbelastung von sich abzuwälzen. Dies ist verständlich, darf aber nicht dazu führen, dass ein Patient hierdurch Nachteile erleidet.

Bezüglich der präoperativen Rasur ist Folgendes erwiesen [5]:

- Die Rasur am Vorabend hat ein signifikant erhöhtes Infektionsrisiko als die OP mit Behaarung. Das Risiko steigt von 0,6 auf $5,6 \%$. Verantwortlich hierfür ist die Tatsache, dass kleinste Hautverletzungen durch die Rasur am Tag danach bakteriell kontaminiert sind (siehe Abb. 4)

- Die Rasur 24 Std. vor der OP hat deutlich höhere Infektraten als die direkt vor OP (7,1 versus 3,1\%)

- Rasur > 24 Std. vor OP bedeutet eine Wundinfektionsrate $>20 \%$

- Chemische Haarentferner haben ein großes Allergiepotenzial und sind somit auch keine wirkliche Lösung

Fasst man all dies zusammen, so sollte das unmittelbare OP-Gebiet erst im Einleitungsraum im OP und unmittelbar vor Beginn der eigentlichen Operation rasiert werden.

\section{Raumluft}

Es gibt keine Daten aus mikrobiologischen Studien, die belegen, dass Luft als relevantes Erregerreservoir bei normalen Operationen angesehen werden kann. Werden allerdings Fremdkörper, z. B. Implantate implantiert, spielt dies eine nicht unerhebliche Rolle. Aus diesem Grunde heraus sollen z.B. in der Endoprothetik die Operationen in OP-Sälen mit LF-Feld durchgeführt werden. Für alle anderen Operationen gibt es keine einheitlichen Empfehlungen der Experten.
Unabhängig davon können Infektionen auf aerogenem Weg zustande kommen. Schwebende Partikel sedimentieren mit dem Luftstrom auf den OP-Situs bzw. den Instrumententisch. Diese Partikel stammen vom OP-Personal, Ärzte wie Pflegekräfte, oder vom Patienten. Insbesondere das körperlich aktive OP-Personal ist aber hier als Streuquelle zu nennen. Jeder Mensch setzt täglich Unmengen von Hautschuppen frei, die oft so klein sind $(14 \mu \mathrm{m}$ Durchmesser), dass sie in der Luft schweben. Mit diesen Hautpartikeln der abgeschilferten Epithelien werden naturgemäß auch Bakterien der Haut mittransportiert [6]. Auch der Nasenrachenraum der Patienten und des OP-Personals ist hier anzuführen, zumal hier häufig pathogenere Keime anzutreffen sind.

Folgende Konsequenzen müssen daher insbesondere bei der Implantatchirurgie hinterfragt werden:

\section{- Mundschutz für Personal und Patient}

Eine immer wieder gern zitierte Studie (siehe Tab. 4) konnte keine positive Wirkung auf die Wundinfektionsrate nachweisen [7]. Es muss aber erwähnt werden, dass diese Studie mit 3088 Operationen nicht an orthopädisch/traumatologischen Patienten durchgeführt wurde. Wie oben diskutiert, ist ja gerade bei der Implantatchirurgie von deutlich höherem Infektrisiko auszugehen. Des Weiteren darf nicht vergessen werden, dass das Weglassen eine extrem hohe Disziplin an alle an der OP Beteiligten stellt. Mikrobiologische Studien haben klar dokumentiert, dass in Abhängigkeit von Gesprächsaktivität die Raten deutlich und signifikant angestiegen sind. Auch erkältetes Personal würde zum potenziellen Problem werden. Zuletzt darf nicht vergessen werden, dass der Mundschutz auch dem Selbstschutz dient. Er schützt das Personal vor Kontamination mit spritzendem Sekret.

Aus diesem Grunde empfehlen wir weiterhin das konsequente Tragen des

\begin{tabular}{lll}
$\begin{array}{l}\text { Tab. } 4 \text { Einfluss einer OP-Maske auf die } \\
\text { Wundinfektionsrate [7] }\end{array}$ & Mit Maske & $\begin{array}{l}\text { Ohne } \\
\text { Maske }\end{array}$ \\
\hline OP Anzahl & 1537 & 1551 \\
Wundinfektrate & $4,7 \%$ & $3,5 \%$
\end{tabular}

Tab. 5 Abdeckung des Instrumententisches [8]

Abdeckung der Instrumente reduziert Komtamination durch Sedimentation aus der Luft um den Faktor 4 (Nebenraum)

- Abdeckung der Instrumente reduziert Komtamination durch Sedimentation aus der Luft um den Faktor 28 (LAF Raum)

- Messbare Sedimentation nur in der Phase der OP Vorbereitung vorhanden

Mundschutzes im OP für das gesamte anwesende Personal und für den Patienten, wenn eine Regionalanästhesie gewählt wurde. Der Mundschutz sollte nach jeder Operation gewechselt werden, Gesprächsaktivität ist auf ein Minimum zu reduzieren.

- Senkung der Aktivität

Die Anzahl von Schwebepartikeln ist direkt abhängig von der Personenzahl, die sich im OP befindet. Aus diesem Grund sollten grundsätzlich nur Personen anwesend sein, die direkt an der OP teilhaben. Alle Aktionen z.B. „Hereinfahren in den $\mathrm{OP}^{\text {“ }}$ sollten ruhig und ohne Bildung größerer Turbulenzen erfolgen.

In einer sehr schönen Studie konnte nachgewiesen werden, dass auch die Abdeckung des Instrumententisches während der präoperativen Phase eine Senkung der Keimbelastung bringt [8]. Diese ist abhängig vom RLT Typ (siehe Tab. 5).

Einflussfaktoren auf den Grad der Luftkontamination:

Personenzahl, Aktivität (erhöht Infektrate) $\rightarrow$ Turbulenz Gespräche (erhöht Infektrate) $\rightarrow$ Streuung pathogener Keime Bear huggers (kein Einfluss)

Morgentliches Aufwischen (erhöht Infektrate) $\rightarrow$ Turbulenz

Offene Türen (erhöht Infektrate) $\rightarrow$ Luftstrom der RLT

\section{Eigenschutz}

Nicht vergessen werden darf, dass der Schutz des Personals den gleichen Stel- 


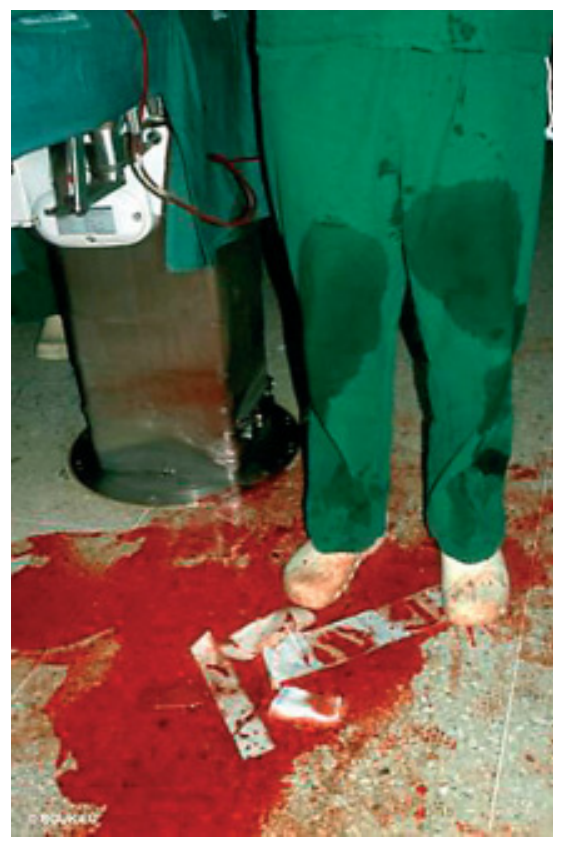

Abb.5 Selbstgefährdung: OP-Kleidung mit Blut durchweicht nach septischem Prothesenausbau.

lenwert haben muss, wie der des Patienten. Die Gefahr sich im OP zu infizieren ist sehr hoch, wenn man nicht Vorsichtsmaßnahmen konsequent durchführt. Hierzu zählen:

- Bekleidung, die eine Durchfeuchtung nicht ermöglicht (siehe Abb. 5)

- 2 Paar Handschuhe. Die Rate von Defekten in den Handschuhen - insbesondere bei Knochenoperationen liegt nach wenigen Minuten bei $80 \%$. Kleine Haarrisse beim OP-Personal an den Händen können zur Hepatitis/ HIV führen. Auch das berufsbedingte Panaritium ist hier anzuführen.

- Visierschutz bei allen Operationen, bei denen mit Blutspritzern zu rechnen ist. Hepatitis und HIV können über die Konjunktiven übertragen werden. Alternativ sind Schutzbrillen zu verwenden.

- Hautpflege ist Pflicht

- Einhalten der Hygienedisziplin bedeutet, jeden Patienten so zu behandeln, als ob er potentiell infektiös sei. Da die Dunkelziffer sehr hoch ist, kann hier nicht genug an die Mitarbeiter appelliert werden

\section{Ausreichendes Fachpersonal}

In Zeiten, in denen die finanziellen Ressourcen sehr limitiert sind, wird natürlich darauf geachtet, Personalkosten einzusparen. Hierbei darf aber nicht vergessen werden, dass kaum jemand unter immer größerem Zeitdruck arbeitet und kon-

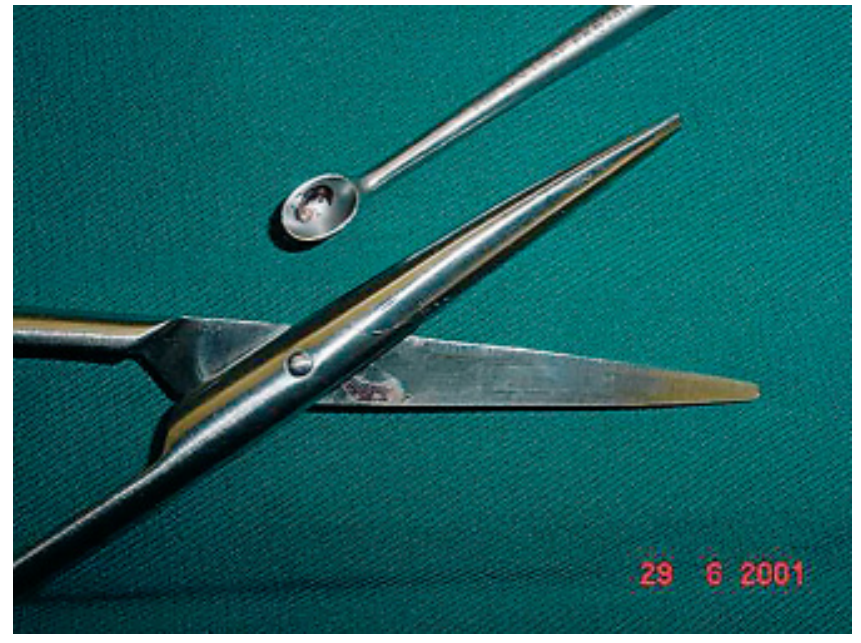

Abb. 6 OP-Instrumente nach der Sterilisation. Blutreste bei unzureichender Aufbereitung. In einem solchen Fall ist das gesamte Sieb abzugeben, da unter den Krusten „biologisches Material“ überlebt.

stant gute Qualität abliefert. Hier ist ein sinnvoller Kompromiss unverzichtbar (siehe Abb. 6). Dies gilt auch für die Bereitstellung einer ausreichenden Zahl von Fachpersonal. Hygiene kostet Geld, erspart aber dem Patienten Leid. Der Klinik werden Kosten durch nicht vergütete Folgeoperationen (DRG-System) und Reduktion von Haftpflichtforderungen eingespart. Das Geld ist gut investiert.

\section{Fazit}

Diese Arbeit kann natürlich das komplexe Gebiet der Hygiene nicht komplett bearbeiten. Nur wesentliche Themenschwerpunkte können diskutiert werden. Natürlich bietet das moderne Gesundheitssystem mit seinen Forderungen nach immer mehr Einsparungen Anlass zur Kritik. Wir dürfen aber nicht verkennen, dass das individuelle Hygieneverhalten jedes Einzelnen im OP-Beschäftigten viel größeren Einfluss auf die Entstehung einer postoperativen Wundinfektion hat, als die Diskussion, welche RLT-Anlage sinnvoll ist. Braucht man für die Entstehung einer Wundinfektion 1000000 Keime, so reduziert sich dies um den Faktor 1000, wenn ein Implantat im Gewebe einliegt. Deshalb müssen gerade in den Fachgebieten, die damit arbeiten, höchste Anforderungen an das Hygienemanagement gestellt werden. Andersherum betrachtet kann eine Klinik, die völlig unterschiedlichen Fachgebieten Zugang zum OP lässt, nicht unterschiedliche Hygieneregime einführen, ein Chaos wäre die Folge. Konsequent betrachtet muss sich deshalb das Hygieneregime jeder Klinik genau an der Fakultät orientieren, die die höchsten Anforderungen der Asepsis benötigt.

Das Schlimmste was passieren kann ist eine Gewöhnung des Personals an eine
Wundinfektion. Jede dieser Komplikationen, auch wenn nur ca. $30 \%$ vermeidbar sind, ist kritisch zu analysieren. Jeder sollte die uns anvertrauten Patienten gerade so behandeln, als wären wir es selbst. Jede Klinik muss ein individuelles Konzept, natürlich orientiert am RobertKoch-Institut (RKI), angepasst an die logistische und bauliche Struktur der Klinik erstellen. An diesen Hygieneplan, dessen zu forderndes Niveau hoch anzusetzen ist, muss sich dann jeder Mitarbeiter halten. Fehlverhalten muss Konsequenzen nach sich ziehen, ansonsten verweicht jede Struktur in kurzer Zeit. Die tägliche Diskussion, ob orientiert an der Literatur individuelle Maßnahmen sinnvoll sind oder nicht, ist kontraproduktiv. Der Hygieneplan sollte solange unverändert bleiben, bis gesicherte neue Erkenntnisse oder Häufung nosokomialer Infektionen dazu zwingen.

Wünschenswert und der Sache dienlich wäre es, wenn die Experten auf dem Gebiet der Hygiene und Mikrobiologie endlich einen gemeinsamen Konsens finden würden. Auch die Diskussion mit den Verwaltungen wäre dann auf fundamentiertem Boden leichter zu führen, da klare einheitliche Vorgaben bestehen würden. Teilweise extrem emotional geführte Diskussionen, die man unter http:// www. uni-uesseldorf.de/WWW/AWMF/ $\mathrm{fg} /$ dakh/st-hygie.htm im Internet nachlesen kann und völlig unterschiedliche Forderungen erstellen, helfen uns im klinischen Alltag nicht weiter.

Der Hygieneplan muss immer für alle gelten. Individuelle Abweichungen Einzelner, die bestimmte Maßnahmen nicht für sinnvoll erachten, dürfen nicht toleriert werden. 


\section{Literatur}

${ }^{1}$ Mutter J, Ebner W, Reichelt A, Rüden $\mathrm{H}$, Daschner F. Sinnvolle und nicht sinnvolle Hygienemaßnahmen in der Orthopädie. Orthopäde 2002; 31: 1039-1044

2 Mäkelä P. Gesunde Haut als Voraussetzung für eine effektive Händedesinfektion. In: Kramer A, Weuffen W, Gröschel D, Heeg P, Hingst V, Lippert H, Rotter M (Hrsg) Klinische Antiseptik. Berlin Heidelberg New York: Springer-Verlag 1993; 97-103

3 Benzer H, Brühl P, Dietzel W, Hartenauer U, Hingst V, Kilian J, Kramer A, Lackner F, Lingnau W, Pauser G, Reybrouck G, Rotter M, Wewalka G (Europäisches interdisziplinäres Komitee für Infektionsprophylaxe, EURIDI$\mathrm{KI}$ ). Meine Hände sind sauber. Warum soll ich sie desinfizieren? Leitfaden zur hygienischen Händedesinfektion. mhp, Wiesbaden 1996
${ }^{4}$ Hayes J. Ring use as a risk factor for hand colonization in a surgical intensive care unit. 41st Annual Interscience conference on antimicroial agents and chemotherapy Dec. 16 19, Chicago,IL, USA, Astract K1333, 2001

5 Seropian et al. Wound infections after preoperative depilatory versus razor preparation. Am J Surg 1971; 121: 251 -254

${ }^{6}$ Noble WC. Dispersal of skin microorganisms. British Journal of Dermatology 1975; 93: 477-485

7 Tunevall TG. Post-operative wound infection and surgical face masks: A controlled study. World J Surg 1991; 15: 383-386

8 Chosky SA, Modha D, Taylor GJS. Optimisation of ultraclean air - the role of instrument preparation. J Bone Joint Surg (Br) 1996; 78: $835-837$
Dr. med. Volkmar Heppert

Leiter der Sektion Posttraumatische Osteitis

\section{Alfons Martens}

OP-Pfleger

OP-Zentrum der BG-Unfallklinik

Ludwigshafen

Prof. Dr. med. Andreas Wentzensen Ärztlicher Direktor

BG-Unfallklinik Ludwigshafen Ludwig-Guttmann-Str. 13

D-67071 Ludwigshafen 